\title{
ORIENTABLE AND NON-ORIENTABLE KLEIN SURFACES WITH MAXIMAL SYMMETRY
}

\author{
by DAVID SINGERMAN
}

(Received 8 September, 1983)

1. Let $X$ be a bordered Klein surface, by which we mean a Klein surface with non-empty boundary. $X$ is characterized topologically by its orientability, the number $k$ of its boundary components and the genus $p$ of the closed surface obtained by filling in all the holes. The algebraic genus $\mathrm{g}$ of $X$ is defined by

$$
g=\left\{\begin{aligned}
2 p+k-1 & \text { if } X \text { is orientable } \\
p+k-1 & \text { if } X \text { is non-orientable. }
\end{aligned}\right.
$$

If $g \geqslant 2$ it is known that if $G$ is a group of automorphisms of $X$ then $|G| \leqslant 12(g-1)$ and that the upper bound is attained for infinitely many values of $g$ ([4], [5]). A bordered Klein surface for which this upper bound is attained is said to have maximal symmetry. A group of $12(g-1)$ automorphisms of a bordered Klein surface of algebraic genus $g$ is called an $M^{*}$-group and it is known that a finite group $G$ is an $M^{*}$-group if and only if it is generated by 3 non-trivial elements $T_{1}, T_{2}, T_{3}$ which obey the relations

$$
T_{1}^{2}=T_{2}^{2}=T_{3}^{2}=\left(T_{1} T_{2}\right)^{2}=\left(T_{2} T_{3}\right)^{3}=I,
$$

Greenleaf and May [2] call the order of $T_{1} T_{3}$ the index of $G$. The index is the order of the group of rotations fixing a boundary component. In [2] it is shown that an $\boldsymbol{M}^{*}$-group acting on two topologically distinct Klein surfaces with the same index must have the same number of boundary components. As they clearly have the same algebraic genus one surface must be orientable and the other surface non-orientable. Greenleaf and May say it would be very interesting to see an actual example of this and in this note we supply one.

2. A method of constructing $M^{*}$-groups of index $q$ is to start with an NEC group $\Gamma$ generated by reflections $c_{1}, c_{2}, c_{3}$ in the sides of a hyperbolic triangle with angles $\pi / 2$, $\pi / 3, \pi / q$ (so that $q>6$ ). A presentation for $\Gamma$ is

$$
\left\{c_{1}, c_{2}, c_{3} \mid c_{1}^{2}=c_{2}^{2}=c_{3}^{2}=\left(c_{1} c_{2}\right)^{2}=\left(c_{2} c_{3}\right)^{3}=\left(c_{1} c_{3}\right)^{a}=1\right\} .
$$

If we consider the Poincare model of the hyperbolic plane as the upper-half complex plane $U$ then $\Gamma$ is a group of Möbius and anti-Möbius transformations. Let $\Gamma^{+}$denote the subgroup of $\Gamma$ of index 2 consisting of the sense-preserving isometries so that $\Gamma^{+}=$ $g p\left\langle c_{1} c_{2}, c_{2} c_{3}\right\rangle$. Now let $G$ be a finite group and $\theta: \Gamma \rightarrow G$ an epimorphism preserving the orders of elements of finite order in $\Gamma$. Then the kernel $K$ of $\theta$ has no elements, besides the identity, of finite order and in particular no reflections. Hence $U / K$ is a compact Klein surface without boundary and we have the following criterion for the orientability of $U / K$.

Glasgow Math. J. 26 (1985) 31-34. 
Proposition 1. The surface $U / K$ is non-orientable if and only if $\theta\left(\Gamma^{+}\right)=G$, (so that $U / K$ is orientable if and only if $\theta\left(\Gamma^{+}\right)$has index 2 in $\left.G\right)$.

Proof. See Theorem 1 of [6].

From the Gauss-Bonnet formula the area of a triangle with angles $\pi / 2, \pi / 3, \pi / q$ is $\pi\left(1-\frac{1}{2}-\frac{1}{3}-1 / q\right)=\pi(q-6) / 6 q$. The order of $G$ can be calculated in terms of the genus $p$ of $S$ (see $5.1,5.2$ of [6] for example) and we find that

$$
\begin{aligned}
|G| & =2 \pi(\eta p-2) / \frac{(q-6) \pi}{6 q} \\
& =\frac{12 q(\eta p-2)}{q-6}
\end{aligned}
$$

where $\eta=1$ if $S$ is non-orientable and $\eta=2$ if $S$ is orientable.

If we put $\theta\left(c_{i}\right)=T_{i},(i=1,2,3)$ then $G$ is generated by the 3 non-trivial elements $T_{1}$, $T_{2}, T_{3}$ obeying the relations $T_{1}^{2}=T_{2}^{2}=T_{3}^{2}=\left(T_{1} T_{2}\right)^{2}=\left(T_{2} T_{3}\right)^{3}=\left(T_{1} T_{3}\right)^{q}=1$ so that $G$ is an $M^{*}$-group of index $q$.

Following [2] we now construct the bordered Klein surface on which $G$ acts as an $M^{*}$-group. On $U$ there is a triangulation consisting of the triangle with angles $\pi / 2, \pi / 3$, $\pi / q$ and all its images under $\Gamma$. This projects onto a triangulation of $S$ consisting of $|G|$ triangles at which $2 q$ meet at a vertex whose angle is $\pi / q$. There are $|G| / 2 q$ such vertices, and these are the fixed points of $T_{1} T_{3}$. If we remove a small topological disc about each of these points we can obtain a bordered Klein surface $S^{*}$ of algebraic genus

$$
g=\eta p+|G| / 2 q-1
$$

admitting $G$ as a group of automorphisms. Using (1) and (2) we obtain

$$
|G|=12(g-1) \text {. }
$$

Thus we have obtained a bordered Klein surface $S^{*}$ with maximal symmetry. Note that the orientability of $S^{*}$ is the same as that of $S$ so that this can be determined using Proposition 1. Hence to construct an example of an $M^{*}$-group acting on both an orientable and a non-orientable surface with the same index we look for a finite group $G$ and two epimorphisms $\theta_{1}: \Gamma \rightarrow G, \theta_{2}: \Gamma \rightarrow G$, preserving the orders of elements of finite order, such that $\theta_{1}\left(\Gamma^{+}\right)=G$ and $\theta_{2}\left(\Gamma^{+}\right)$has index 2 in $G$.

3. To perform such a construction we let $H$ be a simple $M^{*}$-group and $G=C_{2} \times H$. If $H$ is generated by $S_{1}, S_{2}, S_{3}$ with relations including

$$
S_{1}^{2}=S_{2}^{2}=S_{3}^{2}=\left(S_{1} S_{2}\right)^{2}=\left(S_{2} S_{3}\right)^{3}=\left(S_{1} S_{3}\right)^{2 s}=I
$$

and if $C_{2}=g p\left\langle A \mid A^{2}=I\right\rangle$ then $G$ becomes an $M^{*}$-group of index $2 s$ in the following two ways. Firstly, let

$$
T_{1}=\left(I, S_{1}\right), \quad T_{2}=\left(A, S_{2}\right), \quad T_{3}=\left(A, S_{3}\right)
$$


and secondly, let

$$
U_{1}=\left(A, S_{1}\right), \quad U_{2}=\left(A, S_{2}\right), \quad U_{3}=\left(A, S_{3}\right) .
$$

As $T_{1}, T_{2}, T_{3} \in C_{2} \times H$ these elements generate a subgroup $L$ of $C_{2} \times H$. There is an epimorphism $\psi: L \rightarrow H$ by projection onto the second factor, that is $\psi\left(T_{i}\right)=S_{i}, i=1,2,3$. Therefore $|L| \geqslant|H|$ and so $L$ has index 1 or 2 in $C_{2} \times H$. Hence $L \cap(\{I\} \times H)$ has index 1 or 2 in the simple group $\{I\} \times H$ and so the index must be 1 and $L \cap(\{I\} \times H)=\{I\} \times H$ and therefore $L \supseteq\{I\} \times H$. As $\left(A, S_{2}\right) \in L$ we have $L \neq\{I\} \times H$ and so $L=C_{2} \times H$. Therefore $T_{1}, T_{2}, T_{3}$ generate $C_{2} \times H$ and the same argument shows that $U_{1}, U_{2}, U_{3}$ also generate $\mathrm{C}_{2} \times \mathrm{H}$. Furthermore

and

$$
T_{1}^{2}=T_{2}^{2}=T_{3}^{2}=\left(T_{1} T_{2}\right)^{2}=\left(T_{2} T_{3}\right)^{3}=\left(T_{1} T_{3}\right)^{2 s}=I
$$

$$
U_{1}^{2}=U_{2}^{2}=U_{3}^{2}=\left(U_{1} U_{2}\right)^{2}=\left(U_{2} U_{3}\right)^{3}=\left(U_{1} U_{3}\right)^{2 s}=I
$$

so that $C_{2} \times H$ is an $M^{*}$-group in two distinct ways, that is we have two distinct epimorphisms $\theta_{1}: \Gamma \rightarrow G, \theta_{2}: \Gamma \rightarrow G$ defined by $\theta_{1}\left(c_{i}\right)=T_{i},(i=1,2,3)$ and $\theta_{2}\left(c_{i}\right)=U_{i}$, $(i=1,2,3)$.

We now show that $\theta_{1}\left(\Gamma^{+}\right)=G$ and $\theta_{2}\left(\Gamma^{+}\right)$has index 2 in $G$. Let $H_{1}=\{(I, V) \mid V \in H\}$; then $H_{1}$ has index 2 in $G$ and clearly $\theta_{2}\left(\Gamma^{+}\right)=H_{1}$. To show that $\theta_{1}\left(\Gamma^{+}\right)=G$ note that $\theta_{1}\left(c_{1} c_{2}\right) \notin H_{1}$ so that $\theta_{1}\left(\Gamma^{+}\right) \neq H_{1}$. Hence if $\theta_{1}\left(\Gamma^{+}\right)$has index 2 in $G$ then $\theta_{1}\left(\Gamma^{+}\right) \cap H_{1}$ has index 2 in $H_{1}$ which is impossible as $H_{1}$ is a simple $M^{*}$-group.

For $i=1,2$ let $K_{i}$ be the kernel of $\theta_{i}$ and let $X_{i}=U / K_{i}$. Then by proposition $1, X_{1}$ is non-orientable and $X_{2}$ is orientable. Using the ideas of $\$ 2$ we see that we can remove small discs in $X_{1}$ surrounding the fixed points of $T_{1} T_{3}$ to obtain a non-orientable bordered Klein surface $X_{1}^{*}$ admitting $G$ as a maximal group of automorphisms with index $2 s$ and we can remove small discs in $X_{2}$ surrounding the fixed points of $U_{1} U_{3}$ to obtain an orientable bordered Klein surface $X_{2}^{*}$ which also admits $G$ as a maximal group of automorphisms with index $2 s$.

4. We now construct an actual example. We let $2 s=8$ and $G=P S L(2,31)$. Let

$$
X=\left[\begin{array}{rr}
0 & 1 \\
-1 & 0
\end{array}\right], \quad Y=\left[\begin{array}{rr}
3 & -8 \\
-3 & -2
\end{array}\right]
$$

where the matrix entries are regarded as belonging to the field with 31 elements. We calculate that $X, Y$ and $X Y$ have orders 2,3 and 8 in $P S L(2,31)$. From results in [3] we see that $G=g p\langle X, Y\rangle$. (Also see [1] where it is shown that $\operatorname{PSL}(2, q)$ is an image of the triangle group $(2,3,8)$ if and only if $q$ is a prime $\equiv \pm 1 \bmod 16$.)

Now letting

$$
Z=\left[\begin{array}{rr}
14 & 12 \\
12 & -14
\end{array}\right]
$$

we obtain $Z^{2}=(Z X)^{2}=(Z Y)^{2}=I$, so if we let $T_{1}=Z X, T_{2}=Z, T_{3}=Z Y$ we have

$$
T_{1}^{2}=T_{2}^{2}=T_{3}^{2}=\left(T_{1} T_{2}\right)^{2}=\left(T_{2} T_{3}\right)^{3}=\left(T_{1} T_{3}\right)^{8}=I
$$


and hence PSL $(2,31)$ is a simple $M^{*}$-group of index 8 . From $\S 3$ we see that $C_{2} \times$ $P S L(2,31)$ acts as an $M^{*}$-group on both an orientable and non-orientable bordered Klein surface.

We conclude by calculating the topological characteristics of these surfaces. Firstly, from (1) $C_{2} \times P S L(2,31)$ acts as a group of $96(p-1)$ automorphisms (including those which reverse orientation) of a Riemann surface without boundary of topological genus $p$. As $\left|C_{2} \times P S L(2,31)\right|=30 \times 31 \times 32=29,760$ we find that $p=311$. From $\$ 2$ we see that the number of boundary components of the orientable bordered Klein surface we have constructed is $29,760 / 16=1860$ and so its algebraic genus is $2 \times 311+1860-1=2481$. (This is confirmed by $29,760=12 \times 2480$.) Secondly from (1), $C_{2} \times$ PSL $(2,31)$ acts as a group of $48\left(p^{\prime}-2\right)$ automorphisms of a non-orientable Klein surface without boundary of topological genus $p^{\prime}$ and so $p^{\prime}=622$. The number of boundary components of the non-orientable bordered Klein surface we have constructed is again 1860 and so its algebraic genus is $622+1860-1=2481$. Thus $C_{2} \times \operatorname{PSL}(2,31)$ acts as an $M^{*}$-group on both an orientable and non-orientable bordered Klein surface of algebraic genus 2481 with index 8.

I would like to thank Lynne James and Gareth Jones for helpful comments on an earlier draft of this paper and the referee for correcting an error in $\$ 3$.

\section{REFERENCES}

1. B. P. Chetiya, Groups of automorphisms of compact Riemann surfaces. Ph.D. thesis. University of Birmingham, 1971.

2. N. Greenleaf and C. L. May, Bordered Klein surfaces with maximal symmetry. Trans. Amer. Math. Soc. 274 (1982) 265-283.

3. A. M. Macbeath, Generators of the linear fractional groups. Proc. Symp. Pure Math. (Houston, 1967).

4. C. L. May, Automorphisms of compact Klein surfaces with boundary. Pacific J. Math. 59 (1975) 199-210.

5. C. L. May, Large automorphism groups of compact Klein surfaces with boundary. Glasgow Math. J. 18 (1977) 1-10.

6. D. Singerman, Automorphisms of compact non-orientable Riemann surfaces. Glasgow Math. J. 12 (1971) 50-59.

\section{UNIVERSTTY OF SOUTHAMPTON}

